\title{
FIRST ORDER PROPERTIES ON NOWHERE DENSE STRUCTURES
}

\author{
JAROSLAV NEŠETŘIL AND PATRICE OSSONA DE MENDEZ
}

\begin{abstract}
A set $A$ of vertices of a graph $G$ is called $d$-scattered in $G$ if no two $d$-neighborhoods of (distinct) vertices of $A$ intersect. In other words, $A$ is $d$-scattered if no two distinct vertices of $A$ have distance at most $2 d$. This notion was isolated in the context of finite model theory by Gurevich and recently it played a prominent role in the study of homomorphism preservation theorems for special classes of structures (such as minor closed families). This in turn led to the notions of wide, almost wide and quasi-wide classes of graphs. It has been proved previously that minor closed classes and classes of graphs with locally forbidden minors are examples of such classes and thus (relativized) homomorphism preservation theorem holds for them. In this paper we show that (more general) classes with bounded expansion and (newly defined) classes with bounded local expansion and even (very general) nowhere dense classes are quasi wide. This not only strictly generalizes the previous results but it also provides new proofs and algorithms for some of the old results. It appears that bounded expansion and nowhere dense classes are perhaps a proper setting for investigation of wide-type classes as in several instances we obtain a structural characterization. This also puts classes of bounded expansion in the new context. Our motivation stems from finite dualities. As a corollary we obtain that any homomorphism closed first order definable property restricted to a bounded expansion class is a restricted duality.
\end{abstract}

\section{Introduction}

This paper is about special classes of graphs and structures. Typically our classes are countable and contain only finite structures and our motivation is database theory, algorithmic complexity and finite model theory, particularly recently intensively studied homomorphism preservation theorems.

Supported by grant 1M0021620808 of the Czech Ministry of Education and AEOLUS. 
Classical model theory studies properties of abstract mathematical structures (finite or not) expressible in first-order logic [Hod93]. In this context, three classical fundamental preservation theorems have been proved, which connect syntactic and semantic properties of first-order formulas:

- the Łoś-Tarski theorem, which asserts that a first-order formula is preserved under extensions on all structures if, and only if, it is logically equivalent to an existential formula;

- Lyndon's theorem, which asserts that a first-order formula is preserved under surjective homomorphisms on all structures if, and only if, it is logically equivalent to a positive formula [Lyn59];

- the homomorphism preservation theorem which asserts that a firstorder formula is preserved under homomorphisms on all structures if, and only if, it is logically equivalent to an existential-positive formula.

The terms "all structures", which means finite and infinite structures, is crucial in the statement of these theorems.

Finite model theory is the study of first-order logic (and its various extensions) on finite structures [EF96], [Lib04]. In this context, it has been proved that the two first theorems fail when relativized to the finite, that is: there exists a first-order formula that is preserved under extensions on finite structures, but is not equivalent in the finite to an existential formula [Tai59][Gur84][AG97] and there exists a first-order formula that is preserved under surjective homomorphisms on finite structures, but is not equivalent in the finite to a positive formula [AG87][Sto95]. However, a bit surprisingly, the relativized version of the homomorphism preservation theorem to the finite has been recently proved by B. Rossman [Ros07].

Relativizations of homomorphism preservation theorem to specific classes of structures have been studied and in this context A. Atserias and A. Dawar defined classes of graphs called wide, almost wide and quasi-wide (cf. [Daw07a] for instance). (These classes are defined in the Section 3.2.) For instance, it has been proved in [ADG05] that the extension preservation theorem holds in any class $\mathcal{C}$ that is wide, hereditary (i.e. closed under taking substructures) and closed under disjoint unions. Wide classes includes classes with bounded maximum degree. We prove here that an hereditary class of graphs is actually wide if and only if it has a bounded degree (Theorem 3.3).

Also, it has been proved in [ADK04] [ADK06] that the homomorphism preservation theorem holds in any class $\mathcal{C}$ that is almost wide, hereditary 
and closed under disjoint unions. Almost wide classes of graphs include classes of graphs which exclude a minor [KS99]. We characterize almost wide hereditary classes of graphs and prove, in particular, that topologically closed classes of graphs are almost wide.

Dawar [Daw07b] announced that the homomorphism preservation theorem holds in any hereditary quasi-wide class that is closed under disjoint unions. This is a strengthening of the result proved in [ADK04]. Quasiwide classes of graphs include classes of graphs locally excluding a minor [DGK07]. In this paper we prove that any class with bounded expansion is quasi-wide (Theorem 3.19). Bounded expansion classes and algorithmic applications have been introduced in [NOdM05a, NOdM05b, NOdM06a, NOdM06b, NOdM07, NOdM08a, NOdM08b] and have been discussed in Z. Dvořák's PhD thesis [Dvo07a] and in X. Zhu paper [Zhu06].

We shall prove more: we actually give a complete characterization of hereditary classes of graphs which are quasi-wide. This led us to the definition of classes of nowhere dense structures. Classes of nowhere dense structures are defined in this paper (in Section 2.3).

These classes strictly contain all previously studied -in this contextclasses of structures such as classes with bounded local tree width, locally excluded minors, etc, see [Cou90][KS99][ADK04][ADG05][ADK06] [DGK07]; see Fig 2 for the inclusion schema of these classes. Yet we can prove for all classes of nowhere dense structures that the relativized homomorphism preservation theorem holds even for them. Perhaps this also provides a proper setting for this type of questions (about wide, semi-wide and quasiwide classes) and, as we remarked earlier, we obtain characterization theorems.

If a class $\mathcal{K}$ is defined by an existentially positive First Order formula then $\mathcal{K}$ is defined by the existence of a homomorphism from a finite set $\mathcal{F}$ of structures. This in turn means that the complementary class is the class of all structures $\mathbf{A}$ for which there is no homomorphism $\mathbf{F} \longrightarrow \mathbf{A}$ for any $\mathbf{F} \in \mathcal{F}$. This setting is close to (homomorphism ) dualities which were studied recently intensively, [NT00][HN04][NT05].

Combining with the results of [NOdM08c] we prove perhaps surprising fact that any homomorphism closed First Order property when restricted to a Bounded Expansion class is a Restricted Finite Duality. This is stated in Section 4.4 .

This paper is organized as follows: In Section 2 we review all necessary definitions and, among others, we define notions of shallow minor, classes with bounded expansion, classes with bounded local expansion and classes 
of nowhere dense graphs. We also review some background from finite model theory, relational structures and mathematical logic. In the most of this paper we deal with undirected graphs as the difficulty lies there. The relational structures (and hypergraphs in particular) are treated usually by means of Gaifman graph or, sometimes more conveniently, by the incidence graph. (See Sections 2.4 and 4.) However for our purposes this does not suffices and both incidence graphs and Gaifman graphs are too rough tool. Thus in Section 4.1 we find it convenient to define yet another reduction of relational structures (and hypergraphs) to graphs by means of Star Selector.

In Section 3 we prove the characterizations of classes which are wide, almost wide and quasi-wide. This is nontrivial and we need a detail analysis of functions $\Phi_{\mathcal{C}}$ and of its uniform variant $\bar{\Phi}_{\mathcal{C}}$ (which is the key notion for our analysis).

\section{Definitions}

For graphs and, more generally, relational structures, we use standard notation and terminology. In this Section we give the key definitions of this paper.

2.1. Distances, Independence and Scattered Sets. The distance in a graph $G$ between two vertices $x$ and $y$ is the minimum length of a path linking $x$ and $y$ (or $\infty$ if $x$ and $y$ do not belong to the same connected component of $G$ ) and is denoted by $\operatorname{dist}_{G}(x, y)$. Let $G=(V, E)$ be a graph and let $d$ be an integer. The $d$-neighborhood $N_{d}^{G}(u)$ of a vertex $u \in V$ is the subset of vertices of $G$ at distance at most $d$ from $u$ in $G$ : $N_{d}^{G}(u)=\{v \in$ $\left.V: \operatorname{dist}_{G}(u, v) \leq d\right\}$.

Let $r \geq 1$ be an integer. A subset $A$ of vertices of a graph $G$ is $r$ independent if the distance between any two distinct elements of $A$ is strictly greater than $r$. We denote by $\alpha_{r}(G)$ the maximum size of an $r$-independent set of $G$. Thus $\alpha_{1}(G)$ is the usual independence number $\alpha(G)$ of $G$. A subset $A$ of vertices of $G$ is $d$-scattered if $N_{d}^{G}(u) \cap N_{d}^{G}(v)=\emptyset$ for every two distinct vertices $u, v \in A$. Thus $A$ is $d$-scattered if and only if it is $2 r$-independent.

2.2. Shallow minors and Grads. For a graph $G=(V, E)$, we denote by $|G|$ the order of $G$ (that is: $|V|$ ) and by $\|G\|$ the size of $G$ (that is: $|E|$ ).

For any graphs $H$ and $G$ and any integer $d$, the graph $H$ is said to be a shallow minor of $G$ at depth d ([PRS94] attribute this notion, called then low depth minor to Ch. Leiserson and S. Toledo) if there exists a subset $\left\{x_{1}, \ldots, x_{p}\right\}$ of $G$ and a collection of disjoint subsets $V_{1} \subseteq N_{d}^{G}\left(x_{1}\right), \ldots, V_{p} \subseteq$ 


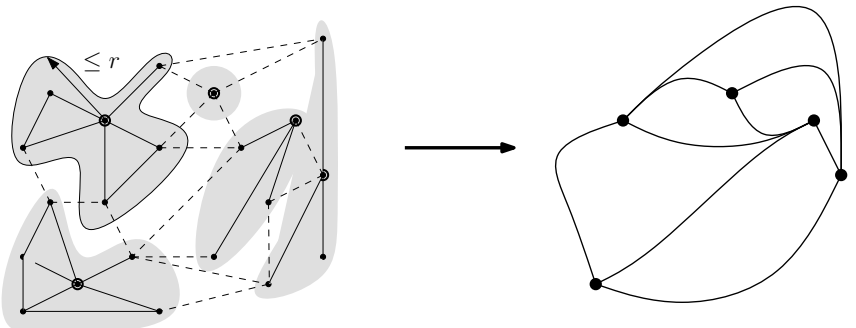

Figure 1. A shallow minor of depth $r$ of a graph $G$ is a simple subgraph of a minor of $G$ obtained by contracting vertex disjoints subgraphs with radius at most $r$

$N_{d}^{G}\left(x_{p}\right)$, each including a connected subgraph of $G$, such that $H$ is a subgraph of the graph obtained from $G$ by contracting each $V_{i}$ into $x_{i}$ and removing loops and multiple edges (see Fig. 1). The set of all shallow minors of $G$ at depth $d$ is denoted by $G \nabla i$. In particular, $G \nabla 0$ is the set of all subgraphs of $G$.

The greatest reduced average density (shortly grad) with rank $r$ of a graph $G$ [NOdM08a, NOdM06a] is defined by formula

$$
\nabla_{r}(G)=\max \left\{\frac{\|H\|}{|H|}: H \in G \nabla r\right\}
$$

By extension, for a class of graphs $\mathcal{C}$, we denote by $\mathcal{C} \nabla i$ the set of all shallow minors at depth $i$ of graphs of $\mathcal{C}$, that is:

$$
\mathcal{C} \nabla i=\bigcup_{G \in \mathcal{C}}(G \nabla i)
$$

Hence we have

$$
\mathcal{C} \subseteq \mathcal{C} \nabla 0 \subseteq \mathcal{C} \nabla 1 \subseteq \cdots \subseteq \mathcal{C} \nabla i \subseteq \ldots
$$

Also, for a class $\mathcal{C}$ of graphs we define the expansion of the class $\mathcal{C}$ as:

$$
\nabla_{i}(\mathcal{C})=\sup _{G \in \mathcal{C}} \nabla_{i}(G)
$$

Notice that $\nabla_{i}(\mathcal{C})=\nabla_{0}(\mathcal{C} \nabla i)$.

2.3. Classes of Graphs. Although almost all results of this paper can be formulated in the "local" form (for a single graph with special properties) we find it useful to formulate our results by means of properties of classes of graphs. 
A class $\mathcal{C}$ of graphs is hereditary if every induced subgraph of a graph in $\mathcal{C}$ to $\mathcal{C}$, and it is monotone of every subgraph of a graph in $\mathcal{C}$ belongs to $\mathcal{C}$. Notice that for every class $\mathcal{C}$, the smallest (inclusion minimal) super-class of $\mathcal{C}$ which is monotone is $\mathcal{C} \nabla 0$.

Class $\mathcal{C}$ has bounded expansion [NOdM08a] if each of the classes $\mathcal{C} \nabla i$ has bounded density:

$$
\begin{aligned}
\mathcal{C} \text { has bounded expansion } & \Longleftrightarrow \forall i \geq 0: \quad \sup _{G \in \mathcal{C} \nabla i} \frac{\|G\|}{|G|}<\infty \\
& \Longleftrightarrow \forall i \geq 0: \quad \nabla_{i}(\mathcal{C})<\infty
\end{aligned}
$$

For an extensive studies of bounded expansion classes we refer the reader to [NOdM08a] [NOdM08b] [NOdM08c] [Dvo07a] [Dvo07b]. We shall add two more types of classes: bounded local expansion and class of nowhere dense graphs.

The class $\mathcal{C}$ has bounded local expansion if the balls of bounded radius of graphs in $\mathcal{C}$ have bounded expansion:

$$
\mathcal{C} \text { has bounded local expansion } \Longleftrightarrow \forall \rho, i \geq 0: \sup _{v \in G \in \mathcal{C}} \nabla_{i}\left(G\left[N_{\rho}^{G}(v)\right]\right)<\infty
$$

As bounded expansion classes strictly contain proper minor closed classes (as classes with constant expansion), bounded local expansion classes generalize classes which locally forbid a minor.

The class $\mathcal{C}$ is a class of nowhere dense graphs if no $\mathcal{C} \nabla i$ contains all finite graphs, that is: if each $\mathcal{C} \nabla i$ has bounded clique number:

$\mathcal{C}$ is a class of nowhere dense graphs $\Longleftrightarrow \forall i \geq 0: \sup _{G \in \mathcal{C} \nabla i} \omega(G)<\infty$.

From an intuitive point of view, classes with bounded expansion correspond to "classes of sparse graphs", classes with bounded local expansion to "classes of locally sparse graphs". For a study of classes of nowhere dense graphs, we refer the reader to our companion paper [NOdM08d]. The inclusion of these classes and of several other types of graph classes is depicted Fig. 2. 


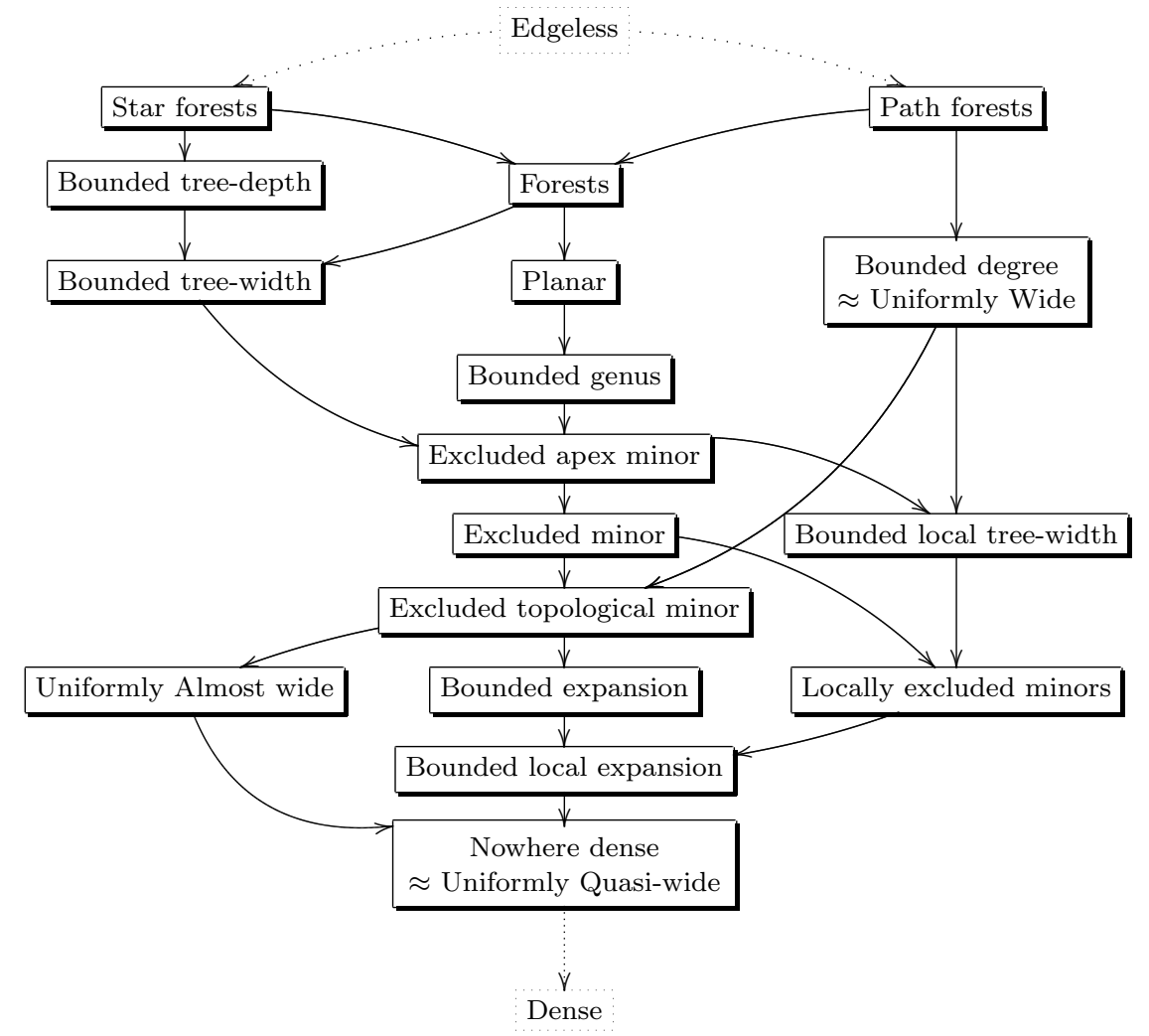

FiguRE 2. Inclusion map of some important properties of classes of graphs.

2.4. Relational Structures and First-Order Logic. Our notation and terminology is standard, see e.g. [EF96]. As we remarked in the introduction most of our paper can be stated for graphs only. The transition to general relational structures (and set-systems) will be explained now.

2.4.1. Relational Structures. A relational vocabulary $\sigma$ is a finite set of relation symbols, each with a specified arity. A $\sigma$-structure A consists of a universe $A$, or domain, and an interpretation which associates to each relation symbol $R \in \sigma$ of some arity $r$, a relation $R^{A} \subseteq A^{r}$. 
A $\sigma$-structure $\mathbf{B}$ is a substructure of $\mathbf{A}$ if $B \subseteq A$ and $R^{B} \subseteq R^{A}$ for every $R \in \sigma$. It is an induced substructure if $R^{B}=R^{A} \cap B^{r}$ for every $R \in \sigma$ of arity $r$. A substructure $\mathbf{B}$ of $\mathbf{A}$ is proper if $\mathbf{A} \neq \mathbf{B}$. If $\mathbf{A}$ is an induced substructure of $\mathbf{B}$, we say that $\mathbf{B}$ is an extension of $\mathbf{A}$. If $\mathbf{A}$ is a proper induced substructure, then $\mathbf{B}$ is a proper extension. If $\mathbf{B}$ is the disjoint union of $\mathbf{A}$ with another $\sigma$-structure, we say that $\mathbf{B}$ is a disjoint extension of $\mathbf{A}$. If $S \subseteq A$ is a subset of the universe of $\mathbf{A}$, then $\mathbf{A} \cap S$ denotes the induced substructure generated by $S$; in other words, the universe of $\mathbf{A} \cap S$ is $S$, and the interpretation in $\mathbf{A} \cap S$ of the $r$-ary relation symbol $R$ is $R^{A} \cap S^{r}$.

(Note that in this paper we shall denote relational structures by boldface letters such as $\mathbf{A}, \mathbf{B}$, and graphs by simple types such as $G, H$.)

2.4.2. First-Order Logic. Let $\sigma$ be a relational vocabulary. The atomic formulas of $\sigma$ are those of the form $R\left(x_{1}, \ldots, x_{r}\right)$, where $R \in \sigma$ is a relation symbol of arity $r$, and $x_{1}, \ldots, x_{r}$ are first-order variables that are not necessarily distinct. Formulas of the form $x=y$ are also atomic.

The collection of first-order formulas is obtained by closing the atomic formulas under negation, conjunction, disjunction, universal and existential first-order quantification. The collection of existential first-order formulas is obtained by closing the atomic formulas and the negated atomic formulas under conjunction, disjunction, and existential quantification. The semantics of first-order logic is standard.

The quantifier rank of a first-order formula is the maximum nesting of quantifiers of its sub-formulas.

Let $\mathbf{A}$ be a $\sigma$-structure, and let $a_{1}, \ldots, a_{n}$ be points in $\mathbf{A}$. If $\phi\left(x_{1}, \ldots, x_{n}\right)$ is a formula with free variables $x_{1}, \ldots, x_{n}$, we denote by $\mathbf{A} \models \phi\left(a_{1}, \ldots, a_{n}\right)$ the fact that $\phi$ is true in $\mathbf{A}$ when $x_{i}$ is interpreted by $a_{i}$. If $m$ is an integer, the first-order $m$-type of $a_{1}, \ldots, a_{n}$ in $\mathbf{A}$ is the collection of all first-order formulas $\phi\left(x_{1}, \ldots, x_{n}\right)$ of quantifier rank at most $m$, up to logical equivalence, for which $\mathbf{A}=\phi\left(a_{1}, \ldots, a_{n}\right)$. If formulas $\phi, \phi^{\prime}$ have the same $m$-type then we write $\phi \equiv^{n} \phi^{\prime}$.

2.4.3. Classes of Structures. The Gaifman graph of a $\sigma$-structure $\mathbf{A}$, denoted by $\mathcal{G}(\mathbf{A})$, is the (undirected) graph whose set of nodes is the universe of $\mathbf{A}$, which is denoted by $A$, and whose set of edges consists of all pairs $\left(a, a^{\prime}\right)$ of distinct elements of $A$ such that $a$ and $a^{\prime}$ appear together in some tuple of a relation in $\mathbf{A}$. This notion coincides with the combinatorial notion of 2-section in the sense of Berge, see e.g. [Ber83]. The degree of a structure is the maximum degree of its Gaifman graph, that is, the maximum number 
of neighbors of nodes of the Gaifman graph. Other notions (such as shallow minor or distance) are defined analogously via Gaifman graphs.

A class $\mathcal{C}$ of relational structures has bounded degree (resp. has bounded expansion, resp. is a class of nowhere dense structures) if the class of graph $\widehat{\mathcal{C}}=\{\mathcal{G}(\mathbf{A}): \mathbf{A} \in \mathcal{C}\}$ has bounded degree (resp. has bounded expansion, resp. is a class of nowhere dense graphs). The class $\mathcal{C}$ is hereditary if any induced substructure of a structure in $\mathcal{C}$ also belongs to $\mathcal{C}$. Notice that a sufficient (but clearly not necessary) condition for $\mathcal{C}$ to be a hereditary class of structures is $\widehat{\mathcal{C}}$ to be a hereditary class of graphs.

Let us remark that alternatively we may convert any relational system and any hypergraph $(X, \mathcal{M})$ to a graph by means of incidence graph $\operatorname{Inc}_{(X, \mathcal{M})}$ which may be defined as the following graph $(V, E)$ where $V=$ $X \cup \mathcal{M}, E=\{(x, e): x \in e \in \mathcal{M}\}$. While the constructions of Gaifman graph and incidence graph lead to similar results, sometimes the incidence graph is preferable (for example in the case of unbounded arities in the hypergraph). The third construction star selector will be introduced in Section 4.1 .

\section{How Wide is a Class?}

3.1. Further Definitions. We find it useful to study wide, almost wide and quasi-wide classes by means of the following functions $\Phi_{\mathcal{C}}$ and $\bar{\Phi}_{\mathcal{C}}$ defined for classes of graphs. It is essential for our approach that we also define the uniform version of these concepts.

Function $\Phi_{\mathcal{C}}$. This function has domain $\mathbb{N}$ and range $\mathbb{N} \cup\{\infty\}$ and $\Phi_{\mathcal{C}}(d)$ is defined for $d \geq 1$ as the minimum $s$ such that the class $\mathcal{C}$ satisfies the following property:

"There exists a function $F: \mathbb{N} \rightarrow \mathbb{N}$ such that for every integer $m$, every graph $G \in \mathcal{C}$ with order at least $F(m)$ contains a subset $S$ of size at most $s$ so that $G-S$ has a $d$-independent set of size $m . "$

We put $\Phi_{\mathcal{C}}(d)=\infty$ if $\mathcal{C}$ does not satisfy the above property for any value of $s$. Moreover, we define $\Phi_{\mathcal{C}}(0)=0$.

Function $\bar{\Phi}_{\mathcal{C}}$. This function has domain $\mathbb{N}$ and range $\mathbb{N} \cup\{\infty\}$ and $\bar{\Phi}_{\mathcal{C}}(d)$ is defined for $d \geq 1$ as the minimum $s$ such that $\mathcal{C}$ satisfies the following property:

"There exists a function $F: \mathbb{N} \rightarrow \mathbb{N}$ such that for every integer $m$, every graph $G \in \mathcal{C}$ and every subset $A$ of vertices 
of $G$ of size at least $F(m)$, the graph $G$ contains a subset $S$ of size at most $s$ so that $A$ includes a $d$-independent set of size $m$ of $G-S . "$

We put $\bar{\Phi}_{\mathcal{C}}(d)=\infty$ if $\mathcal{C}$ does not satisfy the above property for any value of $s$. Moreover, we define $\bar{\Phi}_{\mathcal{C}}(0)=0$.

Notice that obviously $\bar{\Phi}_{\mathcal{C}} \geq \Phi_{\mathcal{C}}$ for every class $\mathcal{C}$ and for every integer $d$.

Definition 3.1. A class of graphs $\mathcal{C}$ is wide (resp. almost wide, resp. quasiwide) if $\Phi_{\mathcal{C}}$ is identically 0 (resp. bounded, resp. finite) [Daw07a]:

$$
\begin{aligned}
\mathcal{C} \text { is wide } & \Longleftrightarrow \forall d \in \mathbb{N}: \Phi_{\mathcal{C}}(d)=0 \\
\mathcal{C} \text { is almost wide } & \Longleftrightarrow \sup _{d \in \mathbb{N}} \Phi_{\mathcal{C}}(d)<\infty \\
\mathcal{C} \text { is quasi-wide } & \Longleftrightarrow \forall d \in \mathbb{N}: \Phi_{\mathcal{C}}(d)<\infty
\end{aligned}
$$

Notice that a hereditary class $\mathcal{C}$ is wide (resp. almost wide, resp. quasiwide) if and only if $\mathcal{C} \nabla 0$ is wide (resp. almost wide, resp. quasi-wide) as deleting edges cannot make it more difficult to find independent sets.

We introduce the following (uniform) variation of Definition 3.1.

Definition 3.2. A class of graphs $\mathcal{C}$ is uniformly wide (resp. uniformly almost wide, resp. uniformly quasi-wide) if $\bar{\Phi}_{\mathcal{C}}$ is identically 0 (resp. bounded, resp. finite):

$$
\begin{aligned}
\mathcal{C} \text { is uniformly wide } & \Longleftrightarrow \forall d \in \mathbb{N}: \bar{\Phi}_{\mathcal{C}}(d)=0 \\
\mathcal{C} \text { is uniformly almost wide } & \Longleftrightarrow \quad \sup _{d \in \mathbb{N}} \bar{\Phi}_{\mathcal{C}}(d)<\infty \\
\mathcal{C} \text { is uniformly quasi-wide } & \Longleftrightarrow \quad \forall d \in \mathbb{N}: \bar{\Phi}_{\mathcal{C}}(d)<\infty
\end{aligned}
$$

Notice that a class $\mathcal{C}$ is uniformly wide (resp. uniformly almost wide, resp. uniformly quasi-wide) if and only if $\mathcal{C} \nabla 0$ is uniformly wide (resp. uniformly almost wide, resp. uniformly quasi-wide) as the property is hereditary in nature and deleting edges cannot make it more difficult to find independent sets.

Based on a construction of Kreidler and Seese [KS99], Atserias et al. [ADK06] proved that if a class excludes a graph minor then it is almost wide. Classes locally excluding a minor have been shown to be quasi-wide by Grohe and Kreutzer [DGK07]. In this paper (in Section 3.5) we characterize these classes.

3.2. Wide classes. As usual we denote by $\Delta(G)$ the maximal degree of a vertex of graph $G$. For a class $\mathcal{C}$ we denote by $\Delta(\mathcal{C})$ the supremum of all 
$\Delta(G)$ for $G \in \mathcal{C}$. Thus $\Delta(\mathcal{C})=\infty$ just means that the graphs in $\mathcal{C}$ may have arbitrary large degrees.

Lemma 3.1. Let $\mathcal{C}$ be a hereditary class of graphs. If $\Delta(\mathcal{C})=\infty$ then $\Phi_{\mathcal{C}}(2)>0$.

Proof. Assume for contradiction that $\mathcal{C}$ satisfies $\Phi_{\mathcal{C}}(2)=0$. Then there exists a function $F: \mathbb{N} \rightarrow \mathbb{N}$ such that every graph $G \in \mathcal{C}$ with order at least $F(2)$ has a 2 -independent set of size 2. As $\Delta(\mathcal{C})=\infty$, the class $\mathcal{C}$ contains a graph $G$ with maximum degree $F(m)$ hence contains the star graph $S_{F(m)}$ as $\mathcal{C}$ is hereditary. Although this graph has order greater than $F(m)$, it contains no 2-independent set of size 2 .

Lemma 3.2. Let $G=(V, E)$ be a graph and let $d, m$ be integers. If $A \subseteq V$ has size at least $\left(\Delta(G)^{d}+1\right) m$ then $A$ includes a d-independent set of size at least $m$.

Proof. Notice that $G^{d}$ has maximum degree at most $\Delta(G)^{d}$ (hence chromatic number at most $\left.\Delta(G)^{d}+1\right)$ and that any independent set of $G^{d}$ is a $d$-independent set of $G$. As at least one color class of $G^{d}$ intersects $A$ on a subset of size at least $|A| / \chi\left(G^{d}\right)$ the lemma follows.

As a consequence of previous lemmas we deduce our first characterization theorem:

Theorem 3.3. Let $\mathcal{C}$ be a hereditary class of graphs. Then the following are equivalent:

- $\Phi_{\mathcal{C}}(2)=0$,

- $\bar{\Phi}_{\mathcal{C}}(2)=0$,

- $\Delta(\mathcal{C})<\infty$,

- $\mathcal{C}$ is wide,

- $\mathcal{C}$ is uniformly wide.

Proof. The theorem follows from the following implications (where the nonobvious implications follow from the two above Lemmas).

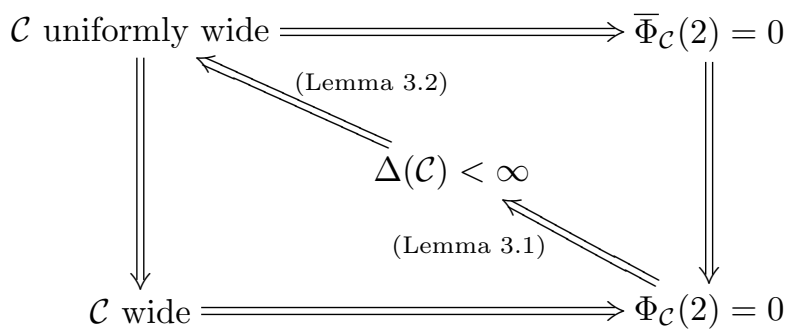



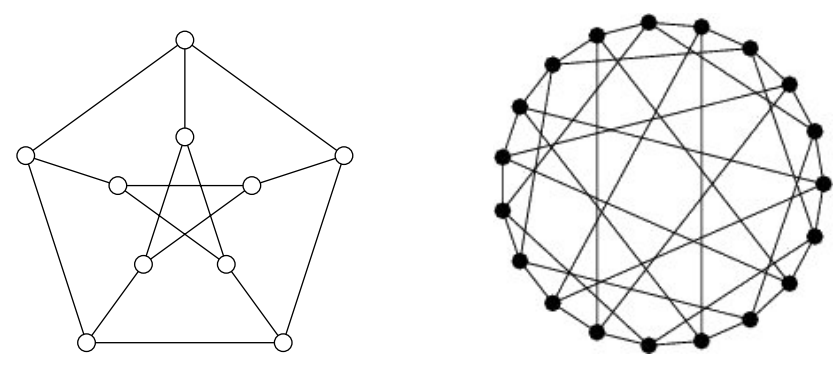

FiguRE 3. Example of graphs whose maximal degree is smaller than their girth

Although the hypothesis that $\mathcal{C}$ is hereditary is not necessary to prove that $\mathcal{C}$ is uniformly wide if and only if $\Delta(\mathcal{C})$ is finite (because the property of being uniformly wide is hereditary in nature), this assumption is necessary in order to prove that a wide class has bounded maximum degree. This is as shown by the next example:

Example 1. Consider the class $\mathcal{C}$ of all 2-connected graphs $G$ satisfying $\Delta(G) \leq \operatorname{girth}(G)$ (some examples of this elusive class of graphs are on Fig.3). Then $\mathcal{C}$ is wide although it does not have a bounded average degree:

Assume that a graph $G \in \mathcal{C}$ has diameter at most $D$. As $G$ is 2-connected, it includes a cycle of length $\operatorname{girth}(G) \leq 2 D$. It follows that $\Delta(G)$ is at most $2 D$ thus $G$ has at most about $(2 D)^{D}$ vertices. Hence for every integer $d$ and $m$, every graph in the class with at least (about) $(2 d m)^{d m}$ vertices has a $d$-independent set of size $m$.

Now we enter a technical part of this paper: In the next three sections we first (in 3.3.1-4) develop techniques for finding $d$-independent sets (for $d=1,2$, even and odd) and then (in 3.3.5) find obstructions to the existence of such sets. This will then lead us (in 3.4) to the estimates for our function $\bar{\Phi}_{\mathcal{C}}$. Finally, in Section 3.5, this will lead to the characterization of almost wide and quasi-wide classes (Theorems 3.20 and 3.25).

3.3. Finding $d$-independent Sets in Graphs. In the following text, $R\left(i_{1}, \ldots, i_{k}\right)$ denotes the $k$-colored Ramsey number, which is the minimum order of a complete graph such that in any $k$-coloration of its edges one finds either a complete graph of order $i_{1}$ colored 1 , or a complete graph of order $i_{2}$ colored $2, \ldots$ or a complete graph of order $i_{k}$ colored $k$. 
3.3.1. Finding 1-independent sets in graphs. The following is a restatement of the Ramsey theorem for graphs. It implies the existence of 1-independent sets in graphs:

Lemma 3.4. Let $G$ be a graph and let $c, n$ be integers. Let $A$ be a subset of at least $R(c, n)$ vertices of $G$. Then either $G$ contains a $K_{c}$ or $A$ includes an independent set of size $n$.

3.3.2. Finding a 2-independent set in a 1-independent set.

Lemma 3.5. Let $G=(A \cup B, E)$ be a bipartite graph and let $p, q, n$ be integers. If $|A| \geq F(p, q, n)$ then at least one of the following properties holds:

- A includes a 2-independent set of size p;

- $A$ includes the principal vertices of a $\dot{K}_{q}$ (the subdivision of $K_{q}$ with every its edge subdivided by exactly one vertex);

- $B$ includes a vertex of degree at least $n$.

Proof. Assume that $B$ includes no vertex of degree at least $n$. Let $k=|B|$ and let $b_{1}, b_{2}, \ldots, b_{k}$ be the vertices in $B$ in any arbitrary order. Let $\Gamma$ be the complete graph with vertex set $A$, whose edges are colored using $\left(\begin{array}{c}n-1 \\ 2\end{array}\right)+1$ colors and which is constructed as follows: We start with $\Gamma$ with vertex set $A$ and without edges. Then we add the edges in $k+1$ steps. At step $i \leq k$ we add to $\Gamma$ all the edges (which are not already been added) between the neighbors of $b_{i}$, coloring them with integers between 1 and $\left(\begin{array}{c}n-1 \\ 2\end{array}\right)$ in such a way that no two edges added at this step get the same color. This is possible as the degree of $b_{i}$ is at most $n-1$. At step $k+1$, we add all the missing edges and assign to them the color $\left(\begin{array}{c}n-1 \\ 2\end{array}\right)+1$.

As $|A| \geq F(p, q, n)$, there exists in $\Gamma$ a monochromatic clique of size $q$ with color in $\left\{1, \ldots,\left(\begin{array}{c}n-1 \\ 2\end{array}\right)\right\}$ or a monochromatic clique of size $p$ with color $\left(\begin{array}{c}n-1 \\ 2\end{array}\right)+1$. If the edges of the clique have color $\left(\begin{array}{c}n-1 \\ 2\end{array}\right)+1$, its vertices define a 2-independent set of $G$ of size $p$. Otherwise, all the edges of the monochromatic clique of size $q$ have been added at different $\left(\begin{array}{l}q \\ 2\end{array}\right)$ steps as they got the same color, hence $G$ includes a $\dot{K}_{q}$ having its principal ("branching") vertices in $A$.

Lemma 3.6. Let $m, a, b, s$ be integers. Let us define inductively the number $\Theta(m, a, b, s)$ by:

$$
\Theta(m, a, b, s)= \begin{cases}F(m, a, b), & \text { if } s=0 \\ F(m, a, b, \Theta(m, a, b, s-1)), & \text { otherwise. }\end{cases}
$$


Let $G=(A \cup B, E)$ be a bipartite graph such that $|A| \geq \Theta(m, a, b, s)$. Then at least one of the following properties holds:

- there exists in $B$ a subset of size at most $s$ whose removal leaves in $A$ a 2-independent set of size $m$;

- A includes all principal vertices of a $\dot{K}_{a}$;

- $B$ includes the $s+1$ vertices of the complete bipartite graph $K_{s+1, b}$.

Proof. We proceed by induction on $s$.

Assume $s=0$. Let $p=m, q=a, n=b$. According to Lemma 3.5, either $A$ includes a 2-independent set of size $m$ of $G$ includes a $\dot{K}_{a}$ with principal vertices in $A$ or $B$ includes a vertex of degree at least $b$ hence $G$ includes a star $K_{1, b}$ with the center of the star in $B$.

Assume that $s>0$ and that the result has been proved for $s-1$. Let $p=m, q=a, n=\Theta(m, a, b, s-1)$. According to Lemma 3.5, either $A$ includes a 2-independent set of size $m$ or $G$ includes a $\dot{K}_{a}$ with principal vertices in $A$ or $B$ includes a vertex of degree at least $\Theta(m, a, b, s-1)$.

In the two first cases we are done thus we may assume that $G$ contains a vertex $v$ of degree at least $\Theta(m, a, b, s-1)$. Let $G^{\prime}$ be the subgraph of $G$ induced by the neighborhood $A^{\prime}$ of $v$ and the set $B^{\prime}$ of the vertices in $B-v$ having at least a neighbor in common with $v$. Then $\left|A^{\prime}\right| \geq \Theta(m, a, b, s-1)$. By induction, either the deletion of a subset $C^{\prime}$ of $s-1$ vertices in $B^{\prime}$ leaves in $A^{\prime}$ of 2-independent set of size $m$ (hence the deletion of the vertices in $C^{\prime} \cup\{v\}$ leaves in $A$ a 2-independent set of size $m$ ) or $G^{\prime}$ includes $K_{s, b}$ with the $s$ vertices in $B^{\prime}$ (thus $G$ includes a $K_{s+1, b}$ with the $s+1$ vertices in $B$ as $v$ is adjacent to all the vertices in $A^{\prime}$ ) or $G^{\prime}$ (hence $G$ ) contains a $\dot{K}_{a}$ with principal vertices in $A^{\prime} \subseteq A$.

Lemma 3.7. Let $G$ be a graph and let $A$ be an independent set of $G$ of order at least $\Theta(m, a, b, s)$. Then at least one of the following properties holds:

- there exists in $G$ a subset of size at most $s$ whose removal leaves in A a 2-independent set of size $m$;

- $G$ includes a $\dot{K}_{a}$ or a $K_{s+1, b}$.

Proof. Consider the bipartite graph $G^{\prime}=\left(A \cup B, E^{\prime}\right)$ where $B$ is the set of all the vertices of $G$ adjacent to a least a vertex in $A$ and $E^{\prime}$ is the subset of the edges of $G$ linking a vertex in $A$ to a vertex in $B$. The result is then a direct consequence of Lemma 3.6.

3.3.3. Finding a $(2 r+1)$-independent set in a $2 r$-independent set. 
Lemma 3.8. Let $G$ be a graph and let $c, n$ be integers. Let $A$ be a $2 r$ independent subset of $G$ of size at least $R(c, n)$. Then either $K_{c} \in G \nabla r$ or $A$ includes $a(2 r+1)$-independent set of size $n$.

Proof. Consider the graph $H \in G \nabla r$ obtained from $G$ by contracting the $r$-neighborhoods of the vertices in $A$ into a set $A^{\prime}$ identified to $A$ (see Fig. 4). According to Lemma 3.4, either $H$ contains a $K_{c}$ (thus $K_{c} \in G \nabla r$ ) or $A^{\prime}$ includes an independent set of size $n$ of $H$, which corresponds to a $(2 r+1)$ independent set of $G$ included in $A$.

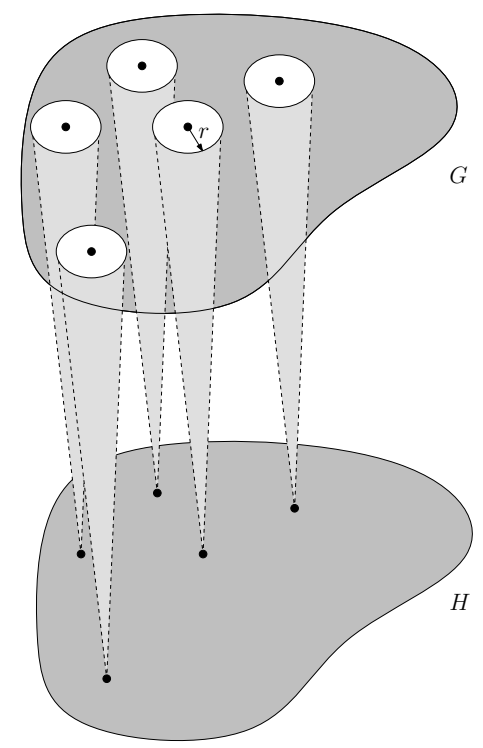

Figure 4 . The reduced graph $H$ is obtained by contracting the $r$-neighborhoods of the vertices in $A$.

3.3.4. Finding a $(2 r+2)$-independent set in a $(2 r+1)$-independent set.

Lemma 3.9. Let $G$ be a graph and let $A$ be a $(2 r+1)$-independent set of $G$ of order at least $\Theta(m, a, b, s)$. Then at least one of the following properties holds:

- there exists in $G$ a subset of size at most $s$ whose removal leaves in A $a(2 r+2)$-independent set of size $m$;

- $G \nabla r$ includes a $\dot{K}_{a}$ or $a K_{s+1, b}$. 
Proof. Consider the graph $H \in G \nabla r$ obtained from $G$ by contracting the $r$-neighborhoods of the vertices in $A$ into a set $A^{\prime}$ identified to $A$ (see Fig. 4). According to Lemma 3.7, either $H$ contains a $\dot{K}_{a}$ or a $K_{s+1, b}$ (thus $\dot{K}_{a} \in$ $G \nabla r$ or $\left.K_{s+1, b} \in G \nabla r\right)$ or $A^{\prime}$ includes a 2-independent set of size $n$ of $H$, which corresponds to a $(2 r+2)$-independent set of $G$ included in $A$.

\subsubsection{Obstructions.}

Lemma 3.10. Let $s$ be an integer. Let $Y$ be a tree of diameter at most $2 r$. Assume the set $L$ of the leaves of $Y$ is partitioned into two sets $A$ and $B$, such that $|B|<s$. Then the deletion of a subset $S$ of at most $s$ vertices of $Y$ cannot leave more than $2 r\left(|B| s+\left(\begin{array}{c}s \\ 2\end{array}\right)\right)<3 r s^{2}$ vertices in the union of the connected components of $Y-S$ having no vertex in $A$.

Proof. Let $S$ be a subset of at most $s$ vertices of $Y$. Root $Y$ at a leaf $t \in A \backslash S$. We order the elements $v_{1}, \ldots, v_{k}$ of this set in such a way that if $v_{i}$ is an ancestor of $v_{j}$ in the tree then $i>j$. Let $b=|B|$. We prove by induction that after the $i$ th vertex deletion, the connected component of $t$ has at most $b+i$ leaves which are not in $A$, and that the number of in the union $X_{i}$ of the vertex sets of the connected components of $Y-v_{1}-\cdots-v_{i}$ having no vertex in $A$ is at most $2 r\left(b i+\left(\begin{array}{c}i \\ 2\end{array}\right)\right)$. For $i=0$ the induction obviously holds. Assume it holds for $0 \leq i<s$. According to the definition of the order of the $v_{i}$ 's, the vertex $v_{i+1}$ belongs to the connected component of $Y-v_{1}-\cdots-v_{i}$ containing $t$. The deletion of $v_{i+1}$ disconnects a collection of sub-trees of this connected component from $t$. The number of leaves of $Y-v_{1}-\cdots-v_{i+1}$ increase at most by one (the father of $v_{i+1}$ in the tree). $X_{i+1}$ is increased by the order of all the sub-trees disconnected from $t$ by $v_{i+1}$ which include no vertex from $A$. This order is bounded by above by the sum of the orders of the paths from $v_{i+1}$ to the leaves of these sub-trees, hence by $2 r(b+i)$. Thus $\left|X_{i+1}\right| \leq 2 r(b+i)+\left|X_{i}\right| \leq 2 r\left(b(i+1)+\left(\begin{array}{c}i+1 \\ 2\end{array}\right)\right)$.

Lemma 3.11. Let $r, s$ be integers, let $G, H$ be graphs such that $H \in G \nabla r$. Assume $H$ is $(s+1)$-connected and let $D=\max _{|X| \leq s} \operatorname{Diam}(H-X)$. Then $G$ has a subgraph $G^{\prime}$ such that $H \in G^{\prime} \nabla r$ and such that the deletion of any $s$ vertices leaves small connected components of total order at most $2 \mathrm{rs}^{2}$ and a big connected component containing all the other vertices and having diameter strictly smaller than $(2 r+1)(D+1)$.

Proof. Let $h$ be the order of $H$. As $H \in G \nabla r$, there are in $G h$ vertices $x_{1}, \ldots, x_{h}$ and $h$ vertex disjoint subgraphs $B_{1}, \ldots, B_{h}$ with centers $x_{1}, \ldots, x_{h}$ and radius at most $r$ such that $H$ is a subgraph of the graph 
obtained from $G$ by deleting all the vertices of out $\bigcup_{i} B_{i}$ and contracting all the $B_{i}$ 's. We denote by $y_{i}$ the vertex of $H$ corresponding to the ball $B_{i}$.

We construct the subgraph $G^{\prime}$ of $G$ as follows: first we delete all the vertices which are not in $\bigcup_{i} B_{i}$. For every adjacent $y_{i}$ and $y_{j}$ in $H$ we keep exactly one edge in $G^{\prime}$ linking a vertex in $B_{i}$ and a vertex in $B_{j}$ (chosen arbitrarily), while for every non-adjacent $y_{i}$ and $y_{j}$ in $H$ we delete all the edges between $B_{i}$ and $B_{j}$. In each $B_{i}$, we keep only the edges of a shortest path tree (i.e. a BFS-tree) from $x_{i}$. Then we iteratively delete any vertex in $B_{i}$ which has degree 1 in $G^{\prime}$.

The so-obtained $G^{\prime}$ is a subgraph of $G$ which is so that $H \in G^{\prime} \nabla r$. Assume we delete in $G^{\prime}$ a subset $X$ of $s$ vertices, $s_{1}$ in $B_{i_{1}}, s_{2}$ in $B_{i_{2}}, \ldots, s_{k}$ in $B_{i_{k}}$ (thus $s_{1}+s_{2}+\cdots+s_{k}=s$ and $k \leq s$ ). According to Lemma 3.10 and as $H$ is $(s+1)$-connected, the order of the connected components of $G^{\prime}$ disconnected from the $B_{i}$ 's (for $i \notin\left\{i_{1}, \ldots, i_{k}\right\}$ by the deletion of the vertices in $S$ is at most $2 r \sum_{i} s_{i}^{2} \leq 2 r s^{2}$ (in the application of Lemma 3.10 to $B_{i_{j}}$, the set $B$ is the set of the leaves linked to some other $B_{i_{j^{\prime}}}$ ).

The upper bound on the diameter of the big connected components presents no particular difficulties.

\subsection{Computing $\bar{\Phi}_{\mathcal{C}}$.}

Lemma 3.12. Let $\mathcal{C}$ be a monotone class of graphs and let $r \geq 0$ be an integer. Assume $\mathcal{C} \nabla r$ contains every $K_{c}(c \in \mathbb{N})$. Then $\Phi_{\mathcal{C}}(2 r+1)=\infty$.

Proof. Assume for contradiction that $\mathcal{C} \nabla r$ contains every $K_{c}(c \in \mathbb{N})$ but $\Phi_{\mathcal{C}}(2 r+1)=s<\infty$. Let $m=2 r s^{2}+2$ and let $N$ be any big integer. As $K_{N} \in \mathcal{C} \nabla r$, according to Lemma 3.11 there exists in $\mathcal{C}$ a graph $G^{\prime}$ such that the deletion of $s$ vertices cannot leave a $(2 r+1)$-independent set of size bigger than $2 r s^{2}+1<m$.

Lemma 3.13. Let $\mathcal{C}$ be a monotone class of graphs and let $r \geq 0$ be an integer. If $\mathcal{C} \nabla r$ contains every $\dot{K}_{a}(a \in \mathbb{N})$. Then $\bar{\Phi}_{\mathcal{C}}(2 r+2)=\infty$.

Proof. Assume for contradiction that $\mathcal{C} \nabla r$ contains every $\dot{K}_{a}(a \in \mathbb{N})$ but $\bar{\Phi}_{\mathcal{C}}(2 r+2)=s<\infty$.

Let $m=$ and let $N$ be any big integer. As $\dot{K}_{N} \in \mathcal{C} \nabla r$, there exists in $\mathcal{C}$ a graph $G$ having $\dot{K}_{N}$ has a depth $r$ minor. According to the proof of Lemma 3.11, this graphs may be chosen with the following structure: the graph $G$ is covered by vertex disjoint induced sub-trees $Y_{i}(1 \leq i \leq N)$ rooted at $x_{i}$ and having height at most $r$, every leaf of $Y_{i}$ is adjacent to some leaf of $Y_{j}$ (with $j \neq i$ ) and for every $i \neq j$ there exists exactly one edge 
between $Y_{i}$ and $Y_{j}$. Let $A=\left\{x_{1}, \ldots, x_{N}\right\}$. By deleting at most $s$ vertices, one leaves at least unmodified a subgraph induced by at least $N-s$ of the $Y_{i}$ 's. As two $x_{i}$ 's in this subgraph have distance at most $2 r+2$, the set $A$ includes no $(2 r+2)$-independent set of size greater than $s+1$.

Lemma 3.14. Let $\mathcal{C}$ be a monotone class of graphs and let $r \geq 0$ be an integer. If $\mathcal{C} \nabla r$ contains every $K_{s, b}(b \in \mathbb{N})$. Then $\bar{\Phi}_{\mathcal{C}}(2 r+2) \geq s$.

Proof. The proof is similar to the one of Lemma 3.13: assume for contradiction that $\mathcal{C} \nabla r$ contains every $K s, b(b \in \mathbb{N})$ but $\bar{\Phi}_{\mathcal{C}}(2 r+2)=s-1$.

Let $m=$ and let $N$ be any big integer. As $\dot{K}_{N} \in \mathcal{C} \nabla r$, there exists in $\mathcal{C}$ a graph $G$ having $K_{s, N}$ has a depth $r$ minor. According to the proof of Lemma 3.11, this graphs may be chosen with the following structure: the graph $G$ is covered by vertex disjoint induced sub-trees $Y_{i}(1 \leq i \leq s)$ and $Y_{j}(1 \leq j \leq N)$, respectively rooted at $x_{i}$ and $x_{j}^{\prime}$, having height at most $r$, every leaf of $Y_{i}$ is adjacent to some leaf of some $Y_{j}^{\prime}$ and for every $1 \leq i \leq s$ and $1 \leq j \leq N$ there exists exactly one edge between $Y_{i}$ and $Y_{j}^{\prime} s$. Let $A=\left\{x_{1}^{\prime}, \ldots, x_{N}^{\prime}\right\}$. By deleting at most $s-1$ vertices, one leaves at least unmodified a subgraph induced by at least $N-s$ of the $Y_{i}^{\prime} s$ and one of $Y_{i}$. As two vertices $x_{i}^{\prime}$ and $x_{j}^{\prime}$ in this subgraph have distance at most $2 r+2$, the set $A$ includes no $(2 r+2)$-independent set of size greater than $s+1$.

Lemma 3.15. Let $\mathcal{C}$ be a hereditary class of graphs and let $r \geq 0$ be an integer.

- If $\omega(\mathcal{C} \nabla r)=\infty$ then $\bar{\Phi}_{\mathcal{C}}(2 r+1)=\Phi_{\mathcal{C}}(2 r+1)=\infty$;

- otherwise, $\Phi_{\mathcal{C}}(2 r+1)=\Phi_{\mathcal{C}}(2 r)$ and $\bar{\Phi}_{\mathcal{C}}(2 r+1)=\bar{\Phi}_{\mathcal{C}}(2 r)$.

Proof. The case where $r=0$ is settle by Lemma 3.4: If $\omega(\mathcal{C} \nabla 0)=\infty$ then $\Phi_{\mathcal{C}}(1)=\bar{\Phi}_{\mathcal{C}}(1)=\infty$; otherwise, $\Phi_{\mathcal{C}}(1)=\bar{\Phi}_{\mathcal{C}}(1)=0=\Phi_{\mathcal{C}}(0)=\bar{\Phi}_{\mathcal{C}}(0)$. So we shall assume $r \geq 1$.

Assume every $K_{c}(c \in \mathbb{N}$ ) belong to $\mathcal{C} \nabla r$ (i.e. $\omega(\mathcal{C} \nabla r)=\infty)$. According to Lemma 3.12, $\Phi_{\mathcal{C}}(2 r+1)=\infty=\bar{\Phi}_{\mathcal{C}}(2 r+1)$. Otherwise, let $c=\omega(\mathcal{C} \nabla r)+$ 1 .

As $\Phi_{\mathcal{C}}$ and $\bar{\Phi}_{\mathcal{C}}$ are non decreasing, if $\Phi_{\mathcal{C}}(2 r)=\infty$ (resp. $\left.\bar{\Phi}_{\mathcal{C}}(2 r)=\infty\right)$ then $\Phi_{\mathcal{C}}(2 r+1)=\Phi_{\mathcal{C}}(2 r)\left(\right.$ resp. $\left.\bar{\Phi}_{\mathcal{C}}(2 r+1)=\infty\right)$.

Let $s=\Phi_{\mathcal{C}}(2 r)$ and let $m$ be an integer. Then every graph $G \in \mathcal{C}$ of order at least $F(R(c, m))$ contains a subset $S$ of at most $s$ vertices such that $\alpha_{2 r}(G-S) \geq R(c, m)$. According to Lemma 3.8, we have $\alpha_{2 r+1}(G-S) \geq m$ as $c>\omega(\mathcal{C} \nabla r)$. Hence $\Phi_{\mathcal{C}}(2 r+1) \leq s$. As $\Phi_{\mathcal{C}}$ is non decreasing, we deduce $\Phi_{\mathcal{C}}(2 r+1)=\Phi_{\mathcal{C}}(2 r)$. 
Let $s=\bar{\Phi}_{\mathcal{C}}(2 r)$ and let $m$ be an integer. Then for every graph $G \in \mathcal{C}$ and every subset $A$ of size at least $F(R(c, m)), G$ contains a subset $S$ of at most $s$ vertices such that $A$ includes a subset $A^{\prime}$ of size $R(c, m)$ which is $2 r$-independent in $G-S$. According to Lemma $3.8, A^{\prime}$ includes a subset $A^{\prime \prime}$ of size $m$ which is $(2 r+1)$-independent in $G-S$ as $c>\omega(\mathcal{C} \nabla r)$. Hence $\bar{\Phi}_{\mathcal{C}}(2 r+1) \leq s$. As $\bar{\Phi}_{\mathcal{C}}$ is non decreasing, we deduce $\bar{\Phi}_{\mathcal{C}}(2 r+1)=\bar{\Phi}_{\mathcal{C}}(2 r)$.

Lemma 3.16. Let $G=(V, E)$ be a graph, let $d \geq 1, m, s$ be integers and let $A$ be a subset of $V$.

Assume there is $S \subseteq V$ and $A^{\prime} \subseteq A$ such that $A^{\prime}$ is $2 d$-independent in $G-S$ and $\left|A^{\prime}\right| \geq m 2^{s}$.

Then there exists $C \subseteq S$ and $A^{\prime \prime} \subseteq A^{\prime}$ such that $A^{\prime \prime}$ is $2 d$-independent in $G-C,\left|A^{\prime \prime}\right|=m$ and $\bar{K}_{|C|, m} \in G \nabla(d-1)$.

Proof. For $v \in A^{\prime}$, let $L_{v}$ be a minimal subset of $S$ such that $N_{d}^{G-L_{v}}(v) \cap$ $\left(S \backslash L_{v}\right)=\emptyset$. Such a set obviously exists (it can be $S$ ). As $S$ has only $2^{s}$ distinct subsets, there exists a subset $A^{\prime \prime} \subset A^{\prime}$ of size $m$ such that $L_{x}=L_{y}$ for every $x, y \in A^{\prime \prime}$ (call this set $C$ ). Let $x, y$ be any two distinct elements of $A^{\prime \prime}$. We have $N_{d}^{G-C}(x) \cap N_{d}^{G-C}(y)=\emptyset$ for otherwise there would exist an $x-y$ path of length at most $2 d$ avoiding $C$ but not $S$ because $A^{\prime \prime}$ is $2 d$ independent in $G-S$ thus some element of $S \backslash C$ would belong to $L_{x}$ or $L_{y}$. Thus $A^{\prime \prime}$ is $2 d$-independent in $G-C$. Moreover, by the minimality of $L_{x}$, every vertex $v \in L_{x}$ is such that $N_{d}^{G-(C-v)}(x) \cap(S \backslash(C-v)) \neq \emptyset$ and more precisely $v \in N_{d}^{G-(C-v)}(x)$. It follows that for every $x \in A^{\prime \prime}$ there exists a tree $Y_{x}$ of depth at most $d$, which leaves are exactly the vertices in $C$ and such that the $Y_{x}$ 's are pairwise internally vertex disjoint. $\mathcal{C} .:$

Define $\beta(\mathcal{C})$ to be supremum of the integers $s$ such that $\left\{K_{s, n}, n \in \mathbb{N}\right\} \subseteq$

Lemma 3.17. Let $\mathcal{C}$ be a class of graphs and let $r \geq 0$ be an integer.

- If $\beta(\mathcal{C} \nabla r)=\infty$ or $\mathcal{C} \nabla r$ includes all the $\dot{K}_{a}(a \in \mathbb{N})$ then $\bar{\Phi}_{\mathcal{C}}(2 r+$ 2) $=\infty$;

- otherwise, $\bar{\Phi}_{\mathcal{C}}(2 r+2)=\beta(\mathcal{C} \nabla r)$.

Proof. If $\beta(\mathcal{C} \nabla r)=\infty$ or $\mathcal{C} \nabla r$ includes all the $\dot{K}_{a}(a \in \mathbb{N})$ then $\bar{\Phi}_{\mathcal{C}}(2 r+2)=$ $\infty$, according to Lemmas 3.13 and 3.14 .

Otherwise, as $\beta(\mathcal{C} \nabla r)<\infty$ we have $\omega(\mathcal{C} \nabla r)<\infty$. It follows (using Lemma 3.15 and induction) that $\bar{\Phi}_{\mathcal{C}}(2 r+1)<\infty$. According to Lemma 3.9, we get $\bar{\Phi}_{\mathcal{C}}(2 r+2)<\infty$. According to Lemma 3.16 we have then $\bar{\Phi}_{\mathcal{C}}(2 r+2) \leq$ $\beta(\mathcal{C} \nabla r)$. According to Lemma 3.14 we have $\bar{\Phi}_{\mathcal{C}}(2 r+2) \geq \beta(\mathcal{C} \nabla r)$. 
Theorem 3.18. Let $\mathcal{C}$ be a class of graphs and let $r \geq 0$ be an integer. Then

- If $\omega(\mathcal{C} \nabla r)=\infty$ then $\bar{\Phi}_{\mathcal{C}}(2 r+1)=\infty$; otherwise, $\bar{\Phi}_{\mathcal{C}}(2 r+1)=$ $\bar{\Phi}_{\mathcal{C}}(2 r)=\beta(\mathcal{C} \nabla(r-1))$.

- If $\beta(\mathcal{C} \nabla r)=\infty$ or $\mathcal{C} \nabla r$ includes all the $\dot{K}_{a}(a \in \mathbb{N})$ then $\bar{\Phi}_{\mathcal{C}}(2 r+$ $2)=\infty$; otherwise, $\bar{\Phi}_{\mathcal{C}}(2 r+2)=\beta(\mathcal{C} \nabla r)$.

Proof. The second item is a direct consequence of Lemma 3.17. The first item is a consequence of both Lemma 3.15 (to prove the finiteness) and also Lemma 3.17 (to get the value, as the obstructions for $2 r$-case would induce big complete minors in $\mathcal{C} \nabla r$ ).

Problem 3.1. Is there a similar characterization for $\Phi_{\mathcal{C}}$ (where $\mathcal{C}$ is a hereditary class of graphs)?

3.5. Almost Wide Classes and Quasi-Wide Classes. The following three characterizations follow from Theorem 3.25 which is presented next. We chose this order to conform to the natural progression of the results.

Theorem 3.19. Let $\mathcal{C}$ be a class with bounded expansion. Then $\bar{\Phi}_{d}(\mathcal{C}) \leq$ $\nabla_{\lfloor d / 2\rfloor-1}(\mathcal{C})$.

Proof. According to Theorem $3.25, \mathcal{C}$ is uniformly quasi-wide, hence $\bar{\Phi}_{\mathcal{C}}(d)<$ $\infty$ for every $d$. According to Theorem $3.18, \bar{\Phi}_{\mathcal{C}}(d)=\beta(\mathcal{C} \nabla(\lfloor d / 2\rfloor-1))$. As $\nabla_{\lfloor d / 2\rfloor-1}(\mathcal{C})=\nabla_{0}(\mathcal{C} \nabla(\lfloor d / 2\rfloor-1)) \geq \sup _{n \in \mathbb{N}} \nabla_{0}\left(K_{\beta(\mathcal{C} \nabla(\lfloor d / 2\rfloor-1)), n}\right)=$ $\beta(\mathcal{C} \nabla(\lfloor d / 2\rfloor-1))$ we conclude.

We have the following characterization of hereditary almost wide classes of graphs:

Theorem 3.20. Let $\mathcal{C}$ be a hereditary class of graphs. Then the following are equivalent:

- $\mathcal{C}$ is almost wide;

- $\mathcal{C}$ is uniformly almost wide;

- There are $s \in \mathbb{N}$ and $t: \mathbb{N} \rightarrow \mathbb{N}$ such that $K_{s, t(r)} \notin \mathcal{C} \nabla r$ (for all $r \in \mathbb{N}$ ).

Proof. If $\mathcal{C}$ is almost wide then the two next items follow from Theorem 3.18. If $\mathcal{C}$ is such that each $\mathcal{C} \nabla r$ excludes some $K_{s, t(r)}$, then it is uniformly quasiwide according to Theorem 3.25 and the bounding of $\bar{\Phi}_{\mathcal{C}}(d)$ then follows from Theorem 3.18. 
To prove that topological closed classes are almost wide, we need the following technical lemma:

Lemma 3.21. Let $G$ be a graph, let $p, r$ be integers and let $t(r)=\left(p\left(\begin{array}{l}p \\ 2\end{array}\right)\right)^{r+2}$. If $K_{\left(\begin{array}{c}p+1 \\ 2\end{array}\right), t(r)} \in G \nabla r$ then $G$ includes $a \leq(8 r+4)$-subdivision of $K_{p}$.

Proof. Let $q=p\left(\begin{array}{l}p \\ 2\end{array}\right)$ and $t(r)=q^{r+1}$, assume $K_{\left(\begin{array}{c}p+1 \\ 2\end{array}\right), t(r)} \in G \nabla r$ and consider a minimal subgraph $H$ of $G$ such that $K_{\left(\begin{array}{c}p+1 \\ 2\end{array}\right), t(r)} \in H \nabla r$. The first part of $K_{\left(\begin{array}{c}p+1 \\ 2\end{array}\right), t(r)}$ we label as follows: $x_{1}, \ldots, x_{p}$ (for the first $p$ vertices) and $z_{\{1,2\}}, \ldots, z_{\{i, j\}}, \ldots, z_{\{p-1, p\}}$ for the $\left(\begin{array}{c}p \\ 2\end{array}\right)$ next ones. The second part of $K_{\left(\begin{array}{c}p+1 \\ 2\end{array}\right), t(r)}$ we label $y_{1}, \ldots, y_{t(r)}$. Then, in $H$, each of these vertices will correspond to rooted trees of height at most $r$ :

- $x_{1}, \ldots, x_{p}$ will correspond to rooted trees $X_{1}, \ldots, X_{p}$;

- $z_{\{1,2\}}, \ldots, z_{\{i, j\}}, \ldots, z_{\{p-1, p\}}$ will correspond to rooted trees $Z_{\{1,2\}}, \ldots$, $\ldots, Z_{\{i, j\}}, \ldots, Z_{\{p-1, p\}}$;

- $y_{1}, \ldots, y_{t(r)}$ will correspond to rooted trees $Y_{1}, \ldots, Y_{t(r)}$.

Moreover, by minimality of $H$, there exists exactly one edge between one vertex of a $X_{i}$ or a $Z_{\{a, b\}}$ and one vertex of a $Y_{j}$, and each leaf of a $X_{i}$ or a $Y_{\{a, b\}}$ is adjacent to a vertex of some $Y_{j}$.

Each $X_{i}$ either include a vertex of degree at least $q$ or $X_{i}$ has order at most $q^{r+1}$ hence at least one vertex of $X_{i}$ is adjacent to at least $t(r) / q^{r+1}=q$ trees $Y_{j}$. For each $i$, we can select $\left(\begin{array}{l}p \\ 2\end{array}\right)$ trees $Y_{k_{i, j}}($ for $j \neq i)$. By construction, we get $p$ spiders (i.e. subdivision of stars) with centers in $X_{1}, \ldots, X_{p}$, the spider with center in $X_{i}$ having its leaves in $Y_{k_{i, j}}$ for $j \neq i$. By assumption, there exists a path from $Y_{k_{i, j}}$ to $Y_{k_{j, i}}$ going through $Z_{\{i, j\}}$. Altogether, we get a $\leq(8 r+4)$-subdivision of $K_{p}$ in $H$ hence in $G$.

We deduce that excluding a topological minor is sufficient to ensure that a class is almost wide:

Theorem 3.22. Let $\mathcal{C}$ be a proper topologically closed class of graphs (i.e. a class of graphs without subdivisions of some fixed graph). Then $\mathcal{C}$ is almost wide.

Proof. Let $\mathcal{C}$ be a proper topologically closed class of graphs. Without loss of generality, we may assume that $\mathcal{C}$ excludes some $K_{N}$ as a topological minor. Assume for contradiction that for every $s \in \mathbb{N}$ there exists $r(s)$ such that for every $t \in \mathbb{N}, K_{s, t} \in \mathcal{C} \nabla r(s)$. Then $K_{\left(\begin{array}{c}N+1 \\ 2\end{array}\right),\left(N\left(\begin{array}{c}N \\ 2\end{array}\right)\right)^{r(N)+2} \in G \nabla r(N) \text { for }}$ some $G \in \mathcal{C}$. According to Lemma 3.21 the graph $G$ includes a subdivision of $K_{N}$, a contradiction. 
Remark 3.23. Uniformly almost wide classes do not need to be topologically closed and not even have bounded local expansion: Consider the class $\mathcal{C}$ of all graphs $G$ satisfying $\Delta(G) \leq \operatorname{girth}(G)$. Then $\mathcal{C}$ is uniformly almost wide although it does not have a bounded average degree: As the class is hereditary, it is sufficient to prove that $\mathcal{C}$ is almost wide. Let $d$ and $m$ be integers. If a graph $G \in \mathcal{C}$ has diameter at least $D=d m$ then $G$ includes a $d$-independent set of size $m$. Otherwise, if $G$ includes a cycle, then this cycle has length girth $(G) \leq 2 D$, hence $\Delta(G) \leq 2 D$ and $G$ has at most about $(2 D)^{D}$ vertices. Otherwise, if $G$ is acyclic, it is a forest, and the deletion of one vertex is sufficient to get a big $d$-independent set. Hence $\mathcal{C}$ is almost wide. Also, let $\mathcal{D}=\left\{G+K_{1}: G \in \mathcal{C}\right\}$. Obviously, $\mathcal{D}$ is also uniformly almost wide but does not have a bounded local expansion.

We may be more precise when $\mathcal{C}$ is actually minor closed:

Theorem 3.24. Let $\mathcal{C}$ be a minor closed class of graphs and let $s$ be an integer. Then the following are equivalent:

- $\mathcal{C}$ is almost wide and $\Phi_{\mathcal{C}}(d)<s$ for every integer $d \geq 2$;

- $\mathcal{C}$ is uniformly almost wide and $\bar{\Phi}_{\mathcal{C}}(d)<s$ for every integer $d \geq 2$;

- $\mathcal{C}$ excludes some graph $K_{s, t}$.

Proof. If $K_{s, N}$ belongs to $\mathcal{C}$ for every $N \in \mathbb{N}$ then $\bar{\Phi}_{\mathcal{C}}(d) \geq \Phi_{\mathcal{C}}(d) \geq \Phi_{\mathcal{C}}(2) \geq$ $s$. Otherwise, according to Theorem 3.18 we have $s>\bar{\Phi}_{\mathcal{C}}(d) \geq \Phi_{\mathcal{C}}(d)$.

Finally, we have the following characterization of quasi-wide classes:

Theorem 3.25. Let $\mathcal{C}$ be a hereditary class of graphs. The following conditions are equivalent:

- $\mathcal{C}$ is quasi-wide;

- $\mathcal{C}$ is uniformly quasi-wide;

- for every integer $d$ there is an integer $N$ such that $K_{N} \notin \mathcal{C} \nabla d$;

- $\mathcal{C}$ is a class of nowhere dense graphs.

Proof. Assume $\mathcal{C}$ is a class of nowhere dense graphs. According to Lemmas $3.4,3.7,3.8$ and 3.9 then $\mathcal{C}$ is uniformly quasi-wide hence quasi-wide. Conversely, if $\mathcal{C}$ is not a class of nowhere dense graphs, then it is not quasiwide according to Lemma 3.12 hence also not uniformly quasi-wide.

Example 2. For a surface $\Sigma$, let $\mathcal{C}_{\Sigma}$ be the class of the graphs which embed on $\Sigma$. It has been proved in [ADK06] that $\mathcal{C}_{\Sigma}$ is almost wide for every surface $\Sigma$ and that $\Phi_{\mathcal{C}_{\Sigma}}(d)$ is at most equal to the order of the smallest clique which does not embed on $\Sigma$. Again, according to Theorem 3.25, the 
class $\mathcal{C}_{\Sigma}$ is uniformly quasi wide. Hence by Theorem 3.18 , we deduce that $\Phi_{\mathcal{C}_{\Sigma}}(d)=\bar{\Phi}_{\mathcal{C}_{\Sigma}}(d)=2$ for every integer $d$, as every $K_{2, n}$ embed on any surface but not every $K_{3, n}$ does.

Remark 3.26. The classes of nowhere dense graphs are very interesting classes in themselves from both algorithmic and structural point of view. They admit a characterization which combine virtually all concepts which were developed for the study of bounded expansion classes and expose them in the new light. It also appears that classes of nowhere dense graphs are a quantitative generalization of bounded expansion classes developed in [NOdM05a, NOdM05b, NOdM06a, NOdM06b, NOdM07, NOdM08a] and [NOdM08b, Dvo07a] and that there is an evidence these classes reach the limit for structural properties. We postpone this to our companion paper [NOdM08d]

\section{Applications}

4.1. Structures. Using incidence (or Gaifman) graph construction the characterization theorems translate almost verbatim to general finite relational structures. Thus for example we have (as an analogy to Theorem 3.3):

Theorem 4.1. Let $\mathcal{C}$ be a hereditary class of structures. Then the class $\mathcal{C}$ is wide iff $\mathcal{C}$ is uniformly wide iff there is a uniform bound to any degree of a vertex of a structure in $\mathcal{C}$.

For a class $\mathcal{C}$ of structures, recall that $\widehat{\mathcal{C}}$ denotes the class of the Gaifman graphs of the structures in $\mathcal{C}$. From Theorem 3.25 and Theorem 3.24 we get:

Theorem 4.2. Let $\mathcal{C}$ be a hereditary class of structures.

- If $\widehat{\mathcal{C}}$ is a class of nowhere dense graphs then $\mathcal{C}$ is uniformly quasiwide;

- Moreover, if there are $s \in \mathbb{N}$ and $t: \mathbb{N} \rightarrow \mathbb{N}$ such that $K_{s, t(r)} \notin \widehat{\mathcal{C}} \nabla r$ (for all $r \in \mathbb{N}$ ), then $\mathcal{C}$ is uniformly almost wide.

However the situation is not so simple and we should aim for more. The fact that the condition that $\widehat{\mathcal{C}}$ should be a class of nowhere dense graphs is not necessary is displayed by the next example:

Example 3. Let $\mathbf{A}_{n}$ be the triple system on $\{1, \ldots, n\}$ with triples $(1, i, j)$ where $1<i<j$ and let $\mathcal{C}=\left\{\mathbf{A}_{n}: n \in \mathbb{N}\right\}$. It is obvious that $\mathcal{C}$ is almost wide (as deleting point 1 disconnects all the structure $\mathbf{A}_{n}$ ) although $\widehat{\mathcal{C}}=\left\{K_{n}: n \in \mathbb{N}\right\}$ is not a class of nowhere dense graphs. 
This example actually shows that Gaifman graphs do not constitute a good approach to determining whether a class of structures is almost wide (resp. quasi-wide) or not. (And that may be one of the first instances of such phenomenon.) Another approach may be used, which will allow to handle class of structures like the one given in Example 3.

Let $\mathbf{A}$ be a structure and let $\operatorname{StarSel}(\mathbf{A})$ denotes the set of all graphs obtained from the incidence graph of $\mathbf{A}$ by contracting exactly one edge at each vertex representing a relation (hence each relation tuple appears as a star in the graph). The graphs in $\operatorname{StarSel}(\mathbf{A})$ we call the star selectors of A. Let $\mathcal{C}$ be a class of structures. A function $\sigma$ mapping each $\mathbf{A} \in \mathcal{C}$ to $\sigma(\mathbf{A}) \in \operatorname{StarSel}(\mathbf{A})$ is called a star selector choice on $\mathcal{C}$. We denote by $\mathfrak{S}(\mathcal{C})$ the set of all the star selector choices on $\mathcal{C}$ and, for $\sigma \in \mathfrak{S}(\mathcal{C})$, we denote by $\sigma(\mathcal{C})$ the class of graphs $\{\sigma(\mathbf{A}): \mathbf{A} \in \mathcal{C}\}$.

Theorem 4.3. Let $\mathcal{C}$ be an infinite class of structures. Then:

- Let $s$ be an integer. Then $\mathcal{C}$ is almost wide (for $s$ ) if and only if there exists $\sigma \in \mathfrak{S}(\mathcal{C})$, such that $\sigma(\mathcal{C})$ is almost wide (for $s$ );

- $\mathcal{C}$ is quasi-wide if and only if there exists $\sigma \in \mathfrak{S}(\mathcal{C})$, such that $\sigma(\mathcal{C})$ is quasi-wide.

Proof. Assume $\mathcal{C}$ is almost wide. Then, there exists $s \in \mathbb{N}$ such that for every $d \in \mathbb{N}$ there exists $N(d)$ such that every structure $\mathbf{A} \in \mathcal{C}$ of order at least $N(d)$ has a set $S$ of at most $s$ points whose deletion leaves in $\mathbf{A}$ a $d$-independent set of size at least $d$. Let $\psi: \mathcal{C} \rightarrow \mathbb{N}$ be defined as follows: for $\mathbf{A} \in \mathcal{C}, \psi(\mathbf{A})$ is the maximum $d \in \mathbb{N}$ such that $\mathbf{A}$ has a subset of at most $s$ points whose deletion leaves in $\mathbf{A}$ a $d$-independent set of size at least $d$. By assumption we have: $\forall \mathbf{A} \in \mathcal{C}$, if $\mathbf{A}$ has order at least $N(d)$ then $\psi(\mathbf{A}) \geq d$. For $\mathbf{A} \in \mathcal{C}$, there exists a subset $S$ at most $s$ points of $\mathbf{A}$ whose deletion leaves in $\mathbf{A}$ a $\psi(\mathbf{A})$-independent set of size at most $\psi(\mathbf{A})$. Let $G_{\mathbf{A}} \in \operatorname{StarSel}(\mathbf{A})$ be a star selector of $\mathbf{A}$ such that for any relation $R$, if $R$ meets $S$ then the star representing $R$ has its center in $S$. (For other tuples we select stars arbitrarily.) Obviously, the deletion in $G_{\mathbf{A}}$ of the vertices corresponding to $S$ leaves in $G_{\mathbf{A}}$ a $\psi(d)$-independent set of size at least $\psi(d)$. Let $\sigma \in \mathfrak{S}(\mathcal{C})$ be such that $\sigma(\mathbf{A})=G_{\mathbf{A}}$ (for every $\mathbf{A} \in \mathcal{C}$ ). Then the class of graphs $\sigma(\mathcal{C})$ is almost wide and $\Phi_{\sigma(\mathcal{C})}(d) \leq s$ for every $d \in \mathbb{N}$.

Conversely, if the deletion of $s$ elements in $\sigma(\mathbf{A})$ leaves a $2 d$-independent set of size $m$ (for some integers $s, d, m$ ), then the deletions of the corresponding $s$ elements in A leaves a $d$-independent set of size $m$ hence $\Phi_{\mathcal{C}}(2 d) \leq \Phi_{\sigma(\mathcal{C})}(d)$. 
Thus, combining both cases, we see that $\sigma(\mathcal{C})$ is almost wide (for $s$ ) if and only if $\mathcal{C}$ is almost wide (for $s$ ). Moreover, the inequality $\Phi_{\mathcal{C}}(2 d) \leq$ $\Phi_{\sigma(\mathcal{C})}(d)$ also shows that $\mathcal{C}$ is quasi-wide if $\sigma(\mathcal{C})$ is quasi-wide. To prove the equivalence for quasi-wide we have to prove the opposite direction.

Assume now $\mathcal{C}$ is quasi-wide. Then there exists functions $f: \mathbb{N} \rightarrow \mathbb{N}$ and $N: \mathbb{N}^{2} \rightarrow \mathbb{N}$ such that for every $d \in \mathbb{N}$ and every $m \in \mathbb{N}$, every $\mathbf{A} \in \mathcal{C}$ of order at least $N(d, m)$ has a subset of size at most $f(d)$ whose deletions leaves in $\mathbf{A}$ a $d$-independent set of size $m$. For $\mathbf{A} \in \mathcal{C}$ and $d \in \mathbb{N}$, let $\zeta_{d}(\mathbf{A})$ be the maximum size of a $d$-independent set we can get in $\mathbf{A}$ by deleting at most $f(d)$ points. Then the assumption that $\mathcal{C}$ is quasi-wide rewrites as

$$
\forall d \in \mathbb{N}: \quad \liminf _{\mathbf{A} \in \mathcal{C}} \zeta_{d}(\mathbf{A})=\infty
$$

Let $\mathbf{A} \in \mathcal{C}$. Then there exists a sequence $S_{1}, S_{2}, \ldots, S_{d}, \ldots$ of subsets of points of $\mathbf{A}$ such that $\left|S_{d}\right| \leq f(d)$ and the deletions of $S_{d}$ leaves in $\mathbf{A}$ a $d$-independent set of size $\zeta_{d}(\mathbf{A})$. Define the graph $G_{\mathbf{A}}$ as follows: for a relation $R$ of $\mathbf{A}$, if there exists $i$ such that $R$ meets $S_{i}$ choose the minimum such $i$ and represent $R$ by a star with center in $S_{i}$; otherwise, represent $R$ by any star. Let $d \in \mathbb{N}$. Delete in $G_{\mathbf{A}}$ the points in $\bigcup_{i=1}^{d} S_{i}$. Then, all the stars corresponding to relations meeting $S_{d}$ have their center removed (because this center has to belong to some $S_{i}$ for $i \leq d$ ) hence this deletion leaves in $G_{\mathbf{A}}$ a $d$-independent set of size at least $\zeta_{d}(\mathbf{A})-\sum_{i=1}^{d-1} f(i)$. Define $\sigma(\mathbf{A})=G_{\mathbf{A}}$ for every $\mathbf{A} \in \mathcal{C}$. Then $\sigma(\mathcal{C})$ is quasi wide (with function $f^{+}(d)=\sum_{i=1}^{d} f(i)$.

Combining this with Theorems 3.20 and 3.25 we obtain the following characterization of classes of almost wide and quasi-wide structures. Advancing this let us call class $\mathcal{C}$ of structures strongly monotone if $\mathcal{C}$ is closed on substructures (i.e. if it is monotone) and in addition if it is closed on taking contractions: For a structure $\mathbf{A}$ and $x \in X(\mathbf{A})$ contraction of vertex $x$ is the structure $\mathbf{A}^{\prime}$ with vertices $X\left(\mathbf{A}^{\prime}\right)=X(\mathbf{A}) \backslash\{x\}$ with signature $\sigma^{\prime} \cup \sigma$ where the relational system $R^{\prime}$ (corresponding to the $k$-nary, $k>1$, relational symbol $R$ ) is $k-1$-ary and consists from all $k$-1-tuples which we obtain from $k$-tuples of $R$ by removing $x$. Contraction is defined by iterating this construction.

Corollary 4.4. Let $\mathcal{C}$ be a strongly monotone class of structures. Then the following are equivalent:

- $\mathcal{C}$ is almost wide;

- $\mathcal{C}$ is uniformly almost wide; 
- There exists $\sigma \in \mathfrak{S}(\mathcal{C})$ and there are $s \in \mathbb{N}$ and $t: \mathbb{N} \rightarrow \mathbb{N}$ such that $K_{s, t(r)} \notin(\sigma(\mathcal{C})) \nabla r($ for all $r \in \mathbb{N})$.

Proof. If $\mathcal{C}$ is almost wide then the two next items follow from Theorem 3.18. If $\mathcal{C}$ is such that each $\mathcal{C} \nabla r$ excludes some $K_{s, t(r)}$, then it is uniformly quasiwide according to Theorem 3.25 and the bounding of $\bar{\Phi}_{\mathcal{C}}(d)$ then follows from Theorem 3.18.

Corollary 4.5. Let $\mathcal{C}$ be a strongly monotone class of graphs. The following conditions are equivalent:

- $\mathcal{C}$ is quasi-wide;

- $\mathcal{C}$ is uniformly quasi-wide;

- There exists $\sigma \in \mathfrak{S}(\mathcal{C})$ such that for every integer d there is an integer $N$ such that $K_{N} \notin \sigma(\mathcal{C}) \nabla d$;

- There exists $\sigma \in \mathfrak{S}(\mathcal{C})$ such that $\sigma(\mathcal{C})$ is a class of nowhere dense graphs.

One could aim for a more explicit characterization of almost wide and quasi-wide classes of structures (with no reference to existence of a special star-selector). This is leading to an interesting combinatorial problems and it will appear elsewhere.

Finally, let us remark that for hypergraphs (i.e. set systems of unrestricted edge sizes) we have analogous results.

4.2. Algorithmic Consequences. Low tree depth colorations (i.e. colorations such that any $i \leq r$ colors induce a subgraph with tree-depth at most $i$ ) may be computed efficiently:

Theorem 4.6 ([NOdM08b]). For every graph $G$ and every integer $r$, a coloration of $G$ using $P_{r}\left(\nabla_{r^{r}}(G)\right)$ colors such that any $i \leq r$ colors induce a subgraph with tree-depth at most $i$ may be computed in $O\left(P_{r}\left(\nabla_{r^{r}}(G)\right) \cdot|G|\right)$ time.

It follows that for input graphs in a class $\mathcal{C}$, counting the isomorphs of a fixed graph, testing whether a graph contains a fixed graph has a subgraph, etc. may be computed in time

$$
\begin{array}{r}
O(n) \quad \text { if } \mathcal{C} \text { is a class with bounded expansion, } \\
n^{1+o(1)} \quad \text { if } \mathcal{C} \text { is a class of nowhere dense graphs, }
\end{array}
$$

where $n$ is the order of the graph (see [NOdM08b]). 
4.3. Caricatures of Structures. It has been proved in [Ros07] (Corollary 6.14) that there exists a function $r$ such that for every integer $n$ and every finite relational structures $\mathbf{A}$ and $\mathbf{B}$ such that $\mathbf{A} \rightleftarrows^{r(n)} \mathbf{B}$ there exists finite relational structures $\widetilde{\mathbf{A}}$ and $\widetilde{\mathbf{B}}$ such that $\mathbf{A} \rightleftarrows \widetilde{\mathbf{A}} \equiv^{n} \widetilde{\mathbf{B}} \rightleftarrows \mathbf{B}$. (Here we write $\mathbf{A} \rightleftarrows \mathbf{B}$ if $\mathbf{A}, \mathbf{B}$ are homomorphic equivalent. We write $\mathbf{A} \rightleftarrows^{r(n)} \mathbf{B}$ if all substructures in $\mathbf{A}$ and $\mathbf{B}$ with homomorphisms equivalent (as sets). Finally we write $\mathbf{A} \equiv{ }^{n} \mathbf{B}$ if $\mathbf{A} \models \phi$ iff $\mathbf{B} \models \phi$ holds for every first-order formula $\phi$ with quantifier rank $n$. See [Ros07] for details and context of these definitions.)

In particular, for every $m$ there exists a function $f$ such that for every structure $\mathbf{A}$ there exists a caricature structure $\mathbf{A}_{p}$ such that $\mathbf{A}_{p}$ has size at most $f(p)$ and $\mathbf{A} \rightleftarrows \widetilde{\mathbf{A}} \equiv{ }^{n} \mathbf{A}_{p}$.

Theorem 4.7. For every class of structures $\mathcal{C}$ and for every integer $p$, there is an algorithm which computes for $\mathbf{A} \in \mathcal{C},|A|=n$, its caricature $\mathbf{A}_{p}$ in time

$$
\begin{aligned}
O(n) & \text { if } \mathcal{C} \text { is a class with bounded expansion, } \\
n^{1+o(1)} & \text { if } \mathcal{C} \text { is a class of nowhere dense structures, }
\end{aligned}
$$

where $n$ is the size of $\mathbf{A}$.

Corollary 4.8. Let $\mathcal{C}$ be a class of structure and let $\phi$ be a first-order property which is preserved in $\mathcal{C}$ under homomorphism equivalence (i.e. $\mathbf{A}=$ $\phi$ and $\mathbf{A} \rightleftarrows \mathbf{B}$ imply $\mathbf{B} \models \phi)$. Then there is an algorithm that checks whether an input structure $\mathbf{A} \in \mathcal{C}$ satisfies $\phi$ or not in time

$$
\begin{aligned}
O(n) & \text { if } \mathcal{C} \text { is a class with bounded expansion, } \\
n^{1+o(1)} & \text { if } \mathcal{C} \text { is a class of nowhere dense structures, }
\end{aligned}
$$

where $n$ is the size of $\mathbf{A}$.

4.4. Homomorphism Preservation Theorem and Restricted Dualities. According to the result announced by Dawar [Daw07b] and to Theorem 3.25, a first-order formula is preserved under homomorphisms on a class $\mathcal{C}$ of nowhere dense structures if, and only if, it is logically equivalent on $\mathcal{C}$ to an existential-positive formula.

Particularly, for every first-order formula $\phi$ preserved under homomorphisms on a class $\mathcal{C}$ with bounded expansion there exists a finite set $\mathcal{F}_{\phi}$ of finite structures such that for every structure $\mathbf{A} \in \mathcal{C}$ holds $\mathbf{F} \not \models \phi$ if and only if $\mathbf{F} \nrightarrow \mathbf{A}$ for every $\mathbf{F} \in \mathcal{F}_{\phi}$. Moreover, according to the results of [NOdM08c], there exists a finite set $\mathcal{D}_{\phi}$ of finite structures such that $\mathbf{F} \nrightarrow \mathbf{A}$ for every $\mathbf{F} \in \mathcal{F}_{\phi}$ if and only if $\mathbf{A} \rightarrow \mathbf{D}$ for some $\mathbf{D} \in \mathcal{D}_{\phi}$. Moreover, $\mathcal{D}_{\phi}$ 
may be chosen in such a way that no $\mathbf{F} \in \mathcal{F}_{\phi}$ has a homomorphism to any $\mathbf{D} \in \mathcal{D}_{\phi}$, that is:

$$
\begin{aligned}
& \forall \mathbf{A} \in \mathcal{C}: \quad \mathbf{F} \models \phi \Longleftrightarrow\left(\exists \mathbf{F} \in \mathcal{F}_{\phi}: \mathbf{F} \rightarrow \mathbf{A}\right) \Longleftrightarrow\left(\forall \mathbf{D} \in \mathcal{D}_{\phi}: \mathbf{A} \nrightarrow \mathbf{D}\right) \\
& \forall(\mathbf{F}, \mathbf{D}) \in \mathcal{F}_{\phi} \times \mathcal{D}_{\phi}: \quad \mathbf{F} \nrightarrow \mathbf{D}
\end{aligned}
$$

This can be put more symbolically as

$$
\begin{aligned}
& \forall \mathbf{A} \in \mathcal{C}: \quad \mathbf{F}=\phi \Longleftrightarrow\left(\mathcal{F}_{\phi} \rightarrow \mathbf{A}\right) \Longleftrightarrow\left(\mathbf{A} \nrightarrow \mathcal{D}_{\phi}\right) \\
& \mathcal{F}_{\phi} \nrightarrow \mathcal{D}_{\phi}
\end{aligned}
$$

Such statements are called restricted finite dualities and the above statement is then rephrased as "every bounded expansion class has all restricted dualities", see [NOdM06c][NOdM06a][NOdM08c].

As a combination of the above we obtain (perhaps suprising) characterization of relativized first order definable subclasses of a class with bounded expansion:

Corollary 4.9. Let $\mathcal{C}$ be a class with bounded expansion. For a homomorphism closed subclass $\mathcal{K}$ of $\mathcal{C}$ are the following statements equivalent:

- $\mathcal{K}$ is the restriction of a first order definable class to $\mathcal{C}$;

- $\mathcal{K}=\{\mathbf{A}: \mathbf{F} \nrightarrow \mathbf{A}$ for every $\mathbf{F} \in \mathcal{F}\}$ for a finite subset $\mathcal{F}$ of $\mathcal{K}$;

- $\mathcal{K}$ is defined by a restricted duality.

This is an analogy of a result for unrestricted dualities on structures, we refer the reader to [NT00, ADK04, Ros07]. The smaller difference is that we do not assume that the class $\mathcal{K}$ is (relativized) CSP class, there are much more dualities, in fact all of them!

Problem 4.1. Can we require that no $\mathbf{D}_{\phi} \in \mathcal{D}_{\phi}$ satisfies $\phi$ ? It is easy to see that this is the case when $\phi$ is an existential-positive formula. However, as the structures in $\mathcal{D}_{\phi}$ don't have to belong to $\mathcal{C}$, the fact that $\phi$ is logically equivalent on $\mathcal{C}$ to an existential-positive formula is not so helpful.

Example 4. It should be noticed that these duality properties fail to be true in general for classes of nowhere dense structures and even for classes of graphs with bounded local tree-width: consider the class $\mathcal{C}$ of the graphs whose girth is strictly bigger than their maximum degree. Then every subgraph of bounded diameter $r$ has either maximum degree at most $r$ (there are finitely many such graphs) or is a tree, hence has bounded tree-width. However, the triangle free graphs in the class do not have bounded chromatic number hence the class $\mathcal{C}$ fails to have all restricted dualities. 


\section{REFERENCES}

[ADG05] A. Atserias, A. Dawar, and M. Grohe. Preservation under extensions on well-behaved finite structures. In Springer-Verlag, editor, 32nd International Colloquium on Automata, Languages and Programming (ICALP), volume 3580 of Lecture Notes in Computer Science, pages 1437-1449, 2005.

[ADK04] A. Atserias, A. Dawar, and P.G. Kolaitis. On preservation under homomorphisms and unions of conjunctive queries. In Proceedings of the twenty-third ACM SIGMOD-SIGACT-SIGART symposium on Principles of database systems, pages 319 - 329. ACM Press, 2004.

[ADK06] A. Atserias, A. Dawar, and P.G. Kolaitis. On preservation under homomorphisms and unions of conjunctive queries. Journal of the ACM, 53:208-237, 2006.

[AG87] M. Ajtai and Y. Gurevich. Monotone versus positive. Journal of the ACM, 34:1004-1015, 1987.

[AG97] N. Alechina and Y. Gurevich. Syntax vs. semantics on nite structures. Structures in Logic and Computer Science, pages 14-33, 1997.

[Ber83] C. Berge. Graphes. Gauthier-Villars, Paris, troisième edition, 1983.

[Cou90] B. Courcelle. The monadic second-order logic of graphs I: recognizable sets of finite graphs. Information Computation, 85:12-75, 1990.

[Daw07a] A. Dawar. Finite model theory on tame classes of structures. In L. Kučera and A. Kučera, editors, Mathematical Foundations of Computer Science 2007, volume 4708 of Lecture Notes in Computer Science, pages 2-12. Springer, 2007.

[Daw07b] A. Dawar. On preservation theorems in finite model theory. Invited talk at the 6th Panhellenic Logic Symposium - Volos, Greece, July 2007.

[DGK07] A. Dawar, M. Grohe, and S. Kreutzer. Locally excluding a minor. In Proc. 22nd IEEE Symp. on Logic in Computer Science, 2007.

[Dvo07a] Z. Dvořák. Asymptotical structure of combinatorial objects. PhD thesis, Charles University, Faculty of Mathematics and Physics, 2007.

[Dvo07b] Z. Dvořák. On forbidden subdivision characterization of graph classes. European Journal of Combinatorics, 2007. (in press).

[EF96] H.-D. Ebbinghaus and J. Flum. Finite Model Theory. Springer-Verlag, 1996.

[Gur84] Y. Gurevich. Toward logic tailored for computational complexity. In M. M. Richter et al., editor, Computation and Proof Theory, Lecture Notes in Mathematics. Springer-Verlag, 1984.

[HN04] P. Hell and J. Nešetřil. Graphs and Homomorphisms, volume 28 of $O x$ ford Lecture Series in Mathematics and its Applications. Oxford University Press, 2004.

[Hod93] W. Hodges. Model Theory. Cambridge University Press, 1993.

[KS99] M. Kreidler and D. Seese. Monadic NP and graph minors. In Computer Science Logic, volume 1584 of Lecture Notes in Computer Science, pages 126-141. Springer, 1999.

[Lib04] L. Libkin. Elements of Finite Model Theory. Springer-Verlag, 2004.

[Lyn59] R.C. Lyndon. Properties preserved under homomorphism. Pacic J. Math., 9:129-142, 1959. 
[NOdM05a] J. Nešetřil and P. Ossona de Mendez. Aspects algorithmiques des classes d'expansion bornée. In L. Esperet, editor, $7^{e}$ Journes Graphes et Algorithmes, volume 1372-05, pages 55-58. LaBRI - Université Bordeaux I, 2005.

[NOdM05b] J. Nešetřil and P. Ossona de Mendez. The grad of a graph and classes with bounded expansion. In André Raspaud and Olivier Delmas, editors, 7th International Colloquium on Graph Theory, volume 22 of Electronic Notes in Discrete Mathematics, pages 101-106. Elsevier, 2005.

[NOdM06a] J. Nešetřil and P. Ossona de Mendez. Linear time low tree-width partitions and algorithmic consequences. In STOC'06. Proceedings of the 38th Annual ACM Symposium on Theory of Computing, pages 391-400. ACM Press, 2006.

[NOdM06b] J. Nešetřil and P. Ossona de Mendez. Low tree-depth partitions of classes with bounded expansion. In Jan K'ara, editor, Midsummer Combinatorial Workshop 2005 and DIMACS, DIMATIA, Rényi Workshop 2005, volume 2006-770 of KAM Series, pages 76-81, 2006.

[NOdM06c] J. Nešetřil and P. Ossona de Mendez. Tree depth, subgraph coloring and homomorphism bounds. European Journal of Combinatorics, 27(6):10221041,2006

[NOdM07] J. Nešetřil and P. Ossona de Mendez. Fraternal augmentations of graphs, coloration and minors. In Proceedings of the Sixth Czech-Slovak International Symposium on Combinatorics, Graph Theory, Algorithms and Applications, volume 28 of Electronic Notes in Discrete Mathematics, pages 223-230, 2007.

[NOdM08a] J. Nešetřil and P. Ossona de Mendez. Grad and classes with bounded expansion I. decompositions. European Journal of Combinatorics, 29(3):760-776, 2008.

[NOdM08b] J. Nešetřil and P. Ossona de Mendez. Grad and classes with bounded expansion II. algorithmic aspects. European Journal of Combinatorics, 29(3):777$791,2008$.

[NOdM08c] J. Nešetřil and P. Ossona de Mendez. Grad and classes with bounded expansion III. restricted graph homomorphism dualities. European Journal of Combinatorics, 29(4):1012-1024, 2008.

[NOdM08d] J. Nešetřil and P. Ossona de Mendez. On nowhere dense graphs. European Journal of Combinatorics, 2008. submitted.

[NT00] J. Nešetřil and C. Tardif. Duality theorems for finite structures (characterizing gaps and good characterizations). Journal of Combinatorial Theory, Series B, 80:80-97, 2000.

[NT05] J. Nešetřil and C. Tardif. Short answers to exponentially long questions: Extremal aspects of homomorphism duality. SIAM Journal of Discrete Mathematics, 19(4):914-920, 2005.

[PRS94] S. Plotkin, S. Rao, and W.D. Smith. Shallow excluded minors and improved graph decomposition. In 5th Symp. Discrete Algorithms. SIAM, 1994.

[Ros07] B. Rossman. Homomorphisms and first-order logic. Journal of the ACM, 2007. submitted. 
[Sto95] A. Stolboushkin. Finite monotone properties. In Proc. 10th IEEE Symp. on Logic in Computer Science, pages 324-330, 1995.

[Tai59] W. Tait. A counterexample to a conjecture of Scott and Suppes. Journal of Symbolic Logic, 24:15-16, 1959.

[Zhu06] X. Zhu. Colouring graphs with bounded generalized colouring number. Discrete Mathematics, 2006. submitted.

Department of Applied Mathematics and Institute of Theoretical Computer Science (ITI), Charles University, Malostranské nám.25, 11800 Praha 1, Czech REPUBLIC

E-mail address: nesetril@kam.ms.mff.cuni.cz

Centre D'Analyse et de Mathématiques Sociales, CNRS, UMR 8557, 54 Bd Raspail, 75006 Paris, France

E-mail address: pom@ehess.fr 